\title{
White Matter Development is Potentially Influenced in Adolescents with Vertically Transmitted HIV Infections: A Tract-Based Spatial Statistics Study
}

\author{
(D). Li, G. Wu, Z. Wen, J. Zhang, H. Lei, X. Gui, and (D)F. Lin
}

O- EBM

\begin{abstract}
BACKGROUND AND PURPOSE: Convergent evidence indicates that HIV is associated with abnormal WM microstructure in adults. However, little is known about whether HIV affects WM development in adolescents. In this study, we used DTI to investigate the integrity of WM microstructure in adolescents with vertically transmitted HIV infections.
\end{abstract}

MATERIALS AND METHODS: Fifteen HIV-positive adolescents with vertically transmitted infections and 26 HIV-negative controls participated in this study. Whole-brain analysis of fractional anisotropy was performed by Tract-Based Spatial Statistics to localize abnormal WM regions between groups. VOI analysis was further performed to explore the changes in diffusivity indices in the regions with fractional anisotropy alterations. Correlation analyses were used to assess the relationship between fractional anisotropy alterations and clinical measures within the HIV-positive group.

RESULTS: Relative to HIV-negative controls, HIV-positive adolescents demonstrated significantly reduced fractional anisotropy in the corpus callosum, superior and posterior corona radiata, frontal and parietal WM, pre-/postcentral gyrus, and superior longitudinal fasciculus. In the affected regions, fractional anisotropy reductions were caused by an increase in radial diffusivity, and no changes were observed in axial diffusivity. Moreover, fractional anisotropy values in the bilateral frontal WM were negatively correlated with the duration of highly active antiretroviral therapy and were positively associated with the age at onset of highly active antiretroviral therapy.

CONCLUSIONS: These findings suggest that early HIV infections may affect WM development, especially in the frontal WM, corpus callosum, and corona radiata in adolescents, which may be associated with hypomyelination and demyelination. Moreover, WM integrity may serve as a potential new treatment target.

ABBREVIATIONS: $A D=$ axial diffusivity; $F A=$ fractional anisotropy; $H A A R T=$ highly active antiretroviral therapy; $M D=$ mean diffusivity; $R D=$ radial diffusivity; TBSS $=$ Tract-Based Spatial Statistics

$\mathbf{H}^{\mathrm{I}}$ IV infections around the world are increasing constantly. ${ }^{1}$ HIV is a neurotrophic virus affecting the cellular immune system through the infection and destruction of CD4 lympho-

Received January 24, 2015; accepted after revision February 25.

From the Departments of Magnetic Resonance Imaging (J.L., G.W., Z.W., J.Z.) and Infectious Diseases (X.G.), Zhongnan Hospital of Wuhan University, Wuhan, China; and National Center for Magnetic Resonance in Wuhan (H.L., F.L.), State Key Laboratory of Magnetic Resonance and Atomic and Molecular Physics, Wuhan Institute of Physics and Mathematics, Chinese Academy of Sciences, Wuhan, China.

Please address correspondence to Guangyao Wu, MD, PhD, Zhongnan Hospital of Wuhan University, No. 169 Donghu Rd, Wuhan 430071, China; e-mail: wuguangy2002@163.com; and Fuchun Lin, PhD, Wuhan Institute of Physics and Mathematics, Chinese Academy of Sciences., West No. 30 Xiao Hong Shan,

Wuhan 430071, China; e-mail: fclin@wipm.ac.cn

This work was partially supported by the Natural Science Foundation of China (Nos. 81171315, 81227902 and 21221064), and National Basic Research Program of China (973 Program) Grant No. 2011CB707802.

-- Indicates open access to non-subscribers at www.ajnr.org

EBM Evidence-Based Medicine Level 2.

http://dx.doi.org/10.3174/ajnr.A4417 cytes, which causes multiple organs, such as the respiratory system, ${ }^{2}$ digestive system, ${ }^{3}$ and CNS, to have relevant illnesses. ${ }^{4}$ Cognitive impairment, HIV-associated dementia, and AIDS dementia complex are commonly observed. ${ }^{5}$

DTI is capable of examining the WM and providing objective parameters that measure the microstructural features nondestructively and noninvasively. ${ }^{6}$ Recent DTI studies have identified abnormalities in the subcortical WM and corpus callosum, despite appearing normal on conventional MRI in HIV-infected adults. $^{7}$ Abnormal WM integrity, such as reduced fractional anisotropy (FA) or increased mean diffusivity (MD), was found in the splenium, ${ }^{8}$ superior longitudinal fasciculus, ${ }^{9}$ anterior and superior corona radiata, ${ }^{10,11}$ and frontal and parietal $\mathrm{WM}^{12}$ in HIVinfected adults.

Similarly, neuroimaging studies have also demonstrated vast WM abnormalities in adolescents with vertically transmitted HIV infection. For example, a voxel-based morphometry study reported WM atrophy in the posterior part of corpus callosum, 
Table 1: Demographic information for subjects in each group

\begin{tabular}{lccc}
\hline & HIV(+) & HIV(-) & $P$ Value \\
\hline Age (yr) & $15.3 \pm 1.3$ & $15.0 \pm 1.6$ & .54 \\
Sex (male/female) & $8: 7$ & $13: 13$ & .84 \\
Education level (yr) & $7.5 \pm 1.2$ & $8.7 \pm 1.9$ & .05 \\
Current CD4 (cells/mL) & $605.8 \pm 345.0$ & NA & NA \\
Age at first HIV treatment (yr) & $9.5 \pm 3.4$ & NA & NA \\
HIV treatment duration $(\mathrm{mo})$ & $68.3 \pm 39.5$ & NA & NA \\
\% Treated at younger than 2 yr & $13.3(n=2)$ & NA & NA \\
Plasma viral load (copies/mL) & $0-50$ & NA & NA \\
MoCA total score & $25.7 \pm 3.8$ & $27.1 \pm 3.1$ & .21 \\
MMSE total score & $25.8 \pm 2.1$ & $27.3 \pm 2.4$ & .05 \\
\hline
\end{tabular}

Note:-NA indicates not applicable or available; MoCa, Montreal Cognitive Assessment; MMSE, Mini-Mental State Examination.

external capsule, and ventral temporal lobe in vertically infected youths (age range, 13-25 years). ${ }^{13}$ Hoare et al ${ }^{14}$ found that vertically HIV-infected children (age range, 8 -12 years) had lower FA and higher MD and radial diffusivity (RD) in the corpus callosum and increased MD in the superior longitudinal fasciculus. Taken together, these findings suggest that abnormal WM integrity may also be present in adolescents (age range, 12-18 years) with vertically transmitted HIV infections. However, previous studies have focused mainly on adults or younger children, and few studies have focused on adolescents with HIV infections. In addition, highly active antiretroviral therapy (HAART) appears to have a positive impact on the WM in the temporal lobe ${ }^{15}$ but with some toxicity. ${ }^{16}$ The effects of HAART in adolescents are also uncertain; this uncertainty may be attributed to high rates of nonadherence $^{17}$ and loss to follow-up. ${ }^{18}$

Therefore, in this study, we used DTI combined with TractBased Spatial Statistics (TBSS; http://fsl.fmrib.ox.ac.uk/fsl /fslwiki/TBSS) analysis to investigate the integrity of WM microstructure in adolescents with vertically transmitted HIV infections undergoing current HAART.

\section{MATERIALS AND METHODS}

\section{Subjects}

We recruited 15 HIV-positive adolescents undergoing combination HAART (mean age, $15.3 \pm 1.3$ years; range, $13-17$ years). The HIV was confirmed by an enzyme-linked immunosorbent assay and western blot. We also recruited 26 age- and sex-matched HIV-negative subjects (mean age, $15.0 \pm 1.6$ years; range, $12-18$ years). All of the HIV-positive adolescents were infected through mother-to-child transmission during pregnancy, delivery, or breastfeeding, and the HIV-negative subjects' fathers or mothers (or both) also had HIV infections. The socioeconomic statuses of the groups and the cultural and ethnic backgrounds of their families were similar. The detailed demographic information and clinical measures are listed in Table 1. All subjects were recruited from the Center of AIDS Prevention and Cure of Zhongnan Hospital, Wuhan University. The inclusion criteria for HIV-infected subjects were HIV acquisition during the fetal or neonatal period, current treatment with HAART, and right-handedness. For the control subjects, the inclusion criteria were the confirmation of HIV-negative status by enzyme-linked immunosorbent assay and right-handedness.

The exclusion criteria for all the subjects included age younger than 12 years or older than 18 years, acute medical illnesses, cur-

\section{Image Acquisition}

rent or past medical or neurologic disorders, psychiatric diseases, mental retardation, current alcohol or substance abuse, HIV encephalopathy and opportunistic infections, MR imaging contraindications, claustrophobia, and metabolic disturbances or other brain diseases (not HIV-related). For control subjects, the exclusion criteria also included severe school difficulties and any chronic medication other than asthma medication. Most of the HIV-infected participants underwent laboratory evaluations, such as plasma CD4 T-cell counts. The CD4 counts ranged from 12 to 1014 cells/mL (average, 605.8 cells $/ \mathrm{mL}$ ). In this study, the patients who were HIVpositive were all undergoing HAART. Thus, all of the plasma viral loads were undetectable ( $0-50$ copies/mL). The Montreal Cognitive Assessment and the Mini-Mental State Examination were used to assess the subjects' cognitive abilities.

The study was approved by the Medical Ethics Committee of Zhongnan Hospital of Wuhan University, and written informed consent was obtained from all participants or their guardians after a complete description of the measurements required for the study.

All subjects were scanned by a 3T MR imaging scanner (Tim Trio; Siemens, Erlangen, Germany). An 8-channel phased array head coil was used with restraining foam pads to minimize head motion and diminish the sounds of the scanner. A single-shot, spinecho EPI technique with alignment of the anterior/posterior commissure plane was performed with the following parameters: $\mathrm{TR}=6000 \mathrm{~ms}, \mathrm{TE}=87 \mathrm{~ms}$, FOV $=24 \times 24 \mathrm{~cm}^{2}$, acquisition matrix $=128 \times 128$ zero-filled to $256 \times 256$, section thickness $=$ $3 \mathrm{~mm}$ without gap, sections $=45$, number of repetitions $=4$, parallel acceleration factor $=2$. The diffusion sensitizing gradients were applied along 20 noncollinear gradient encoding directions with $b=1000 \mathrm{~s} / \mathrm{mm}^{2}$ with an acquisition without diffusionweighting $\left(b=0 \mathrm{~s} / \mathrm{mm}^{2}\right)$.

\section{Data Processing}

DTI data analysis was performed by the FMRIB Diffusion Toolbox http://www.fmrib.ox.ac.uk/fsl/fdt/index.html). The diffusion-weighted volumes were first aligned to their corresponding non-diffusion-weighted images to minimize image distortion and reduce simple head motion. The diffusion tensor for each voxel was then assessed, and the diffusion tensor was diagonalized to obtain its 3 pairs of eigenvalues $\left(\lambda_{1}, \lambda_{2}, \lambda_{3}\right)$ and eigenvectors. Four diffusion indices, including FA, $\mathrm{MD}$, axial diffusivity $\left(\mathrm{AD}=\lambda_{1}\right)$, and $\mathrm{RD}\left[\mathrm{RD}=\left(\lambda_{2}+\lambda_{3}\right) / 2\right]$ were calculated. These measurements are associated with the microstructural integrity of the WM and are often applied to infer the structural characteristics of the local tissue environment. ${ }^{19}$

Voxelwise, observer-independent, whole-brain analysis of FA images was performed by TBSS. ${ }^{20}$ In brief, the FA volumes for all subjects were first normalized to the Montreal Neurological Institute space. The registered FA images were then averaged to 


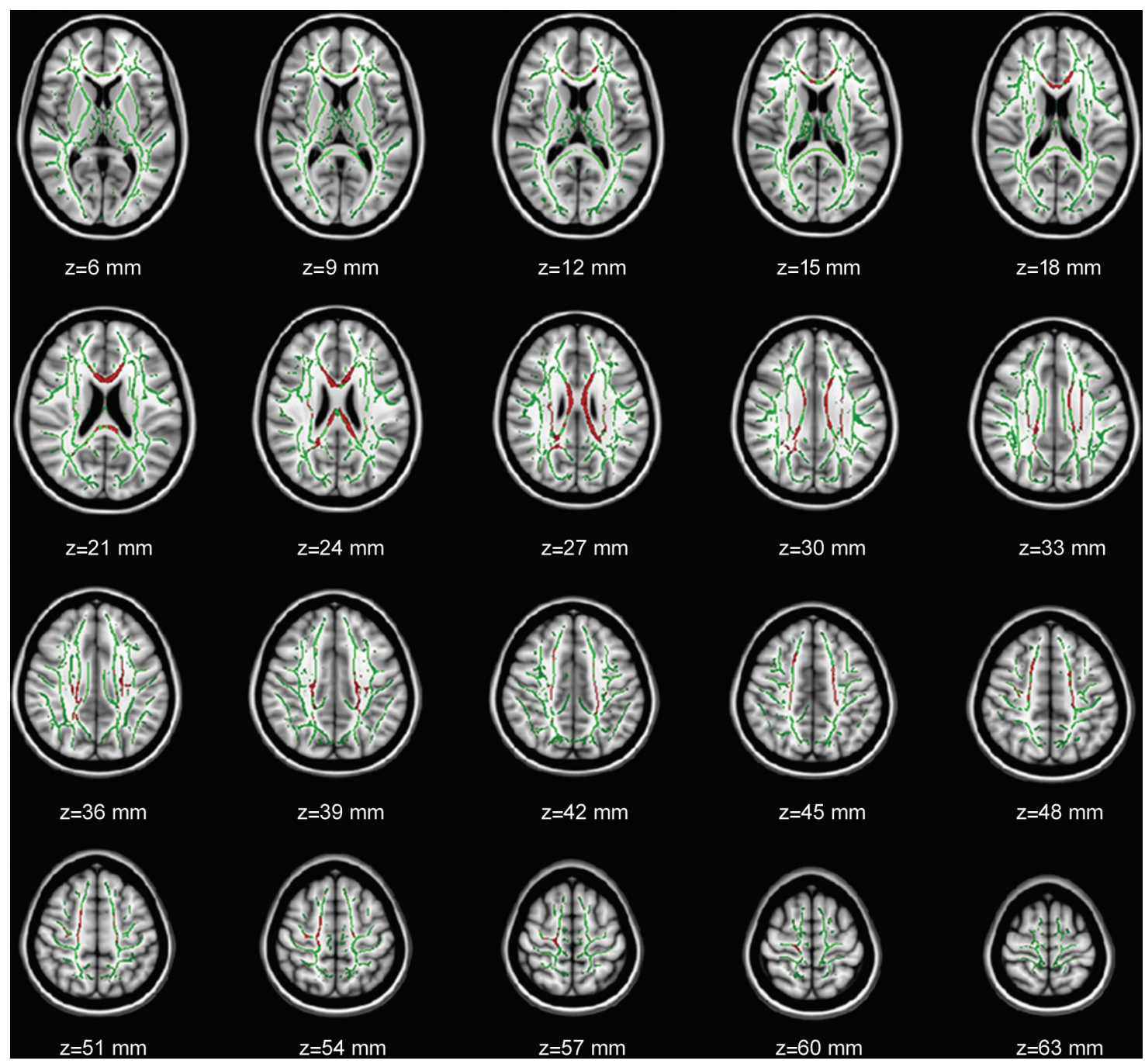

FIG 1. TBSS analysis of fractional anisotropy maps. Areas in red are brain regions where FA is significantly reduced $(P<.05$, corrected by multiple comparison) in HIV-positive subjects relative to HIV-negative controls. The results are shown overlaid on the Montreal Neurological Institute 152-T1 template and the mean FA skeleton (green). The left side of the image corresponds to the right hemisphere of the brain. FA changes occur in the region with Montreal Neurological Institute coordinates in the $\mathrm{z}$-direction between $\mathrm{z}=6 \mathrm{and} \mathrm{z}=63$.

obtain a mean FA image, and the mean FA image was applied to create a mean FA skeleton, which represents the main fiber tracts. The mean FA skeleton was further thresholded to exclude gray matter and CSF by a value of 0.2 . Following this step, the aligned FA data for each subject were projected onto the mean skeleton to create a skeletonized FA map. To identify FA differences between groups, we fed the skeletonized FA data into the voxel-by-voxel, nonparametric statistical analysis with age, sex, and educational levels as covariates. Threshold-free cluster enhancement was used to obtain the significant differences between 2 groups at $P<.05$ after accounting for multiple comparisons by controlling for the family-wise error rate. The significant results were located with the JHU-ICBM-DTI-81 WM label atlas (http://cmrm.med. jhmi.edu/) in the Montreal Neurological Institute space.

To explore mechanisms related to the FA changes, we further performed VOI analysis to investigate alterations in diffusivity indices ( $\mathrm{AD}, \mathrm{RD}$, and $\mathrm{MD}$ ) in the regions with FA alterations. The VOI mask was first extracted on the basis of the clusters with intergroup FA differences and were then inversely transformed to the original images of each subject. The mean values of the diffusivity indices were calculated. A 1-way analysis of covariance with the group as the independent variable and diffusivity indices as the dependent variables was performed (controlling for age, sex, and education levels). A significance level of $P<.05$ (Bonferroni correction for multiple comparisons) was used.

Multiple linear regression analysis was performed to investigate whether there were relationships between the clinical variables and FA changes in the affected regions with WM abnormalities. A $P<.05$ (uncorrected) was considered significant.

\section{RESULTS}

\section{Demographic Information}

Table 1 lists the detailed demographic and clinical information for the HIV-positive adolescents and HIV-negative controls. The HIV-positive subjects demonstrated no significant differences in age, sex, or education level (in years). There were no differences in the Montreal Cognitive Assessment or Mini- 
Mental State Examination total scores between these 2 study groups. The average CD4 count in HIV-positive subjects was $605.8 \pm 345.0$ cells $/ \mathrm{mL}$.

\section{TBSS Results}

The spatial distribution of the brain regions demonstrating decreased FA in the HIV-positive adolescents is presented in Fig 1 and Table 2. Compared with the controls, the HIV-positive adolescents demonstrated significantly reduced FA in the bilateral corpus callosum (including the genu, body, and splenium), bilateral superior and posterior corona radiata, bilateral frontal and parietal WM, bilateral pre-/postcentral gyrus, and left superior longitudinal fasciculus. In no regions did HIV-positive adolescents demonstrate higher FA than controls.

\section{VOI Results}

To investigate the mechanisms related to the FA changes, the brain regions with significantly decreased FA were extracted for VOI-based analyses of other diffusion indices. Twelve of the 14 VOIs demonstrated significantly increased $\mathrm{RD}(P<.05$

Table 2: Neuroanatomic regions with decreased FA in HIV-positive subjects relative to HIVnegative controls ${ }^{a}$

\begin{tabular}{llrrrr}
\hline & & \multicolumn{3}{c}{ MNI Coordinates } & \\
\cline { 3 - 4 } \multicolumn{1}{c}{ Anatomic Region } & Hemisphere & \multicolumn{1}{c}{ X } & \multicolumn{1}{c}{ Y } & Z & $\begin{array}{c}\text { Cluster Size } \\
\left(\mathbf{m m}^{3}\right)\end{array}$ \\
\hline Genu of corpus callosum & Bilateral & -13 & 20 & 24 & 530 \\
Body of corpus callosum & Bilateral & -11 & 16 & 24 & 2228 \\
Splenium & Bilateral & -11 & -35 & 24 & 564 \\
Superior corona radiata & Left & -20 & -25 & 39 & 497 \\
Superior corona radiata & Right & 20 & -24 & 37 & 386 \\
Posterior corona radiata & Left & -21 & -28 & 34 & 262 \\
Posterior corona radiata & Right & 25 & -27 & 33 & 249 \\
Frontal WM & Left & -18 & 5 & 43 & 164 \\
Frontal WM & Right & 17 & 19 & 44 & 399 \\
Pre-/postcentral gyrus WM & Left & -21 & -25 & 42 & 269 \\
Pre-/postcentral gyrus WM & Right & 21 & -24 & 42 & 524 \\
Parietal WM & Left & -22 & -31 & 40 & 149 \\
Parietal WM & Right & 17 & -54 & 28 & 437 \\
SLF & Left & -30 & -17 & 34 & 85 \\
\hline
\end{tabular}

Note:-MNI indicates Montreal Neurological Institute; SLF, superior longitudinal fasciculus.

${ }^{a} P<.05$, threshold-free cluster enhancement multiple comparison-corrected. Coordinates for the peak voxels are displayed. after Bonferroni correction). The other 2 VOIs trended toward increased RD. No significant differences were detected in $\mathrm{AD}$ in any of the VOIs. Only 3 VOIs had significantly increased MD $(P<.05$ after Bonferroni correction). The results are listed in Table 3.

\section{Correlation Results}

Using a multiple linear regression analysis, we found that in HIVinfected adolescents, the FA values of the bilateral WM were negatively correlated with the duration of HAART (right frontal WM: $r=-0.634, P=.011$, Fig $2 A$; left frontal WM: $r=-0.623, P=$ .013 , Fig $2 B$ ), and the FA values of the bilateral WM were positively correlated with the age at onset of HAART (right frontal WM: $r=0.615, P=.015$, Fig $2 C$; left frontal WM: $r=0.553, P=$ .032 , Fig 2D).

\section{DISCUSSION}

In this study, we used DTI to explore the integrity of WM microstructure in adolescents with vertically transmitted HIV infections by voxelwise TBSS analysis. HIV-positive adolescents showed decreased FA in the bilateral corpus callosum, bilateral superior and posterior corona radiata, bilateral frontal and parietal WM, bilateral pre-/postcentral gyrus, and left superior longitudinal fasciculus. These results reflect a disruption in the microstructure of the WM in HIV-infected adolescents. VOI analysis demonstrated that decreased FA in the HIV-positive subjects was mainly a result of increased $\mathrm{RD}$ but no changes in $\mathrm{AD}$; these findings were perhaps a manifestation of the disrupted integrity of myelin. Moreover, a multiple regression analysis demonstrated that the FA values in the bilateral frontal WM were negatively correlated with the duration of HAART and positively correlated with the age at onset of HAART.

The findings that FA values within

Table 3: Group differences in diffusivity indices from VOls

\begin{tabular}{|c|c|c|c|c|c|c|c|c|c|c|}
\hline \multirow[b]{2}{*}{ Anatomic Region } & \multirow[b]{2}{*}{ Hemisphere } & \multicolumn{3}{|c|}{$\operatorname{AD}\left(\times 10^{-3} \mathrm{~mm}^{2} / \mathrm{s}\right)$ (Mean) } & \multicolumn{3}{|c|}{$\mathrm{RD}\left(\times 10^{-3} \mathrm{~mm}^{2} / \mathrm{s}\right)$ (Mean) } & \multicolumn{3}{|c|}{$\mathrm{MD}\left(\times 10^{-3} \mathrm{~mm}^{2} / \mathrm{s}\right)$ (Mean) } \\
\hline & & HIV- & HIV+ & $\begin{array}{c}P \\
\text { Value }\end{array}$ & HIV- & HIV+ & $\begin{array}{c}P \\
\text { Value }\end{array}$ & HIV- & HIV+ & $\begin{array}{c}P \\
\text { Value }\end{array}$ \\
\hline Genu of corpus callosum & Bilateral & $1.62 \pm 0.08$ & $1.58 \pm 0.08$ & .16 & $0.36 \pm 0.03$ & $0.40 \pm 0.06$ & .004 & $0.78 \pm 0.03$ & $0.79 \pm 0.05$ & .22 \\
\hline Body of corpus callosum & Bilateral & $1.78 \pm 0.06$ & $1.78 \pm 0.06$ & .99 & $0.41 \pm 0.05$ & $0.49 \pm 0.07$ & $.0003^{\mathrm{a}}$ & $0.87 \pm 0.04$ & $0.92 \pm 0.05$ & $.002^{\mathrm{a}}$ \\
\hline Splenium & Bilateral & $1.66 \pm 0.06$ & $1.67 \pm 0.05$ & .41 & $0.33 \pm 0.03$ & $0.37 \pm 0.04$ & $.0003^{\mathrm{a}}$ & $0.77 \pm 0.02$ & $0.80 \pm 0.03$ & $.001^{\mathrm{a}}$ \\
\hline Superior corona radiata & Left & $1.21 \pm 0.04$ & $1.20 \pm 0.03$ & .33 & $0.53 \pm 0.02$ & $0.56 \pm 0.04$ & $.0003^{\mathrm{a}}$ & $0.76 \pm 0.02$ & $0.78 \pm 0.03$ & .01 \\
\hline Superior corona radiata & Right & $1.26 \pm 0.05$ & $1.26 \pm 0.04$ & .78 & $0.49 \pm 0.02$ & $0.53 \pm 0.03$ & $.00009^{a}$ & $0.74 \pm 0.02$ & $0.77 \pm 0.02$ & $.001^{\mathrm{a}}$ \\
\hline Posterior corona radiata & Left & $1.23 \pm 0.06$ & $1.23 \pm 0.03$ & .81 & $0.59 \pm 0.03$ & $0.62 \pm 0.03$ & $.002^{\mathrm{a}}$ & $0.80 \pm 0.03$ & $0.82 \pm 0.02$ & .02 \\
\hline Posterior corona radiata & Right & $1.26 \pm 0.04$ & $1.25 \pm 0.03$ & .32 & $0.57 \pm 0.03$ & $0.61 \pm 0.04$ & $.002^{\mathrm{a}}$ & $0.80 \pm 0.03$ & $0.82 \pm 0.04$ & .05 \\
\hline Frontal WM & Left & $1.35 \pm 0.07$ & $1.32 \pm 0.05$ & .22 & $0.44 \pm 0.03$ & $0.48 \pm 0.04$ & $.001^{\mathrm{a}}$ & $0.74 \pm 0.03$ & $0.76 \pm 0.03$ & .09 \\
\hline Frontal WM & Right & $1.37 \pm 0.06$ & $1.32 \pm 0.07$ & .02 & $0.40 \pm 0.03$ & $0.48 \pm 0.03$ & $.0003^{\mathrm{a}}$ & $0.72 \pm 0.03$ & $0.73 \pm 0.03$ & .21 \\
\hline Pre-/postcentral gyrus WM & Left & $1.32 \pm 0.06$ & $1.29 \pm 0.06$ & .14 & $0.47 \pm 0.03$ & $0.50 \pm 0.03$ & $.0007^{\mathrm{a}}$ & $0.75 \pm 0.02$ & $0.76 \pm 0.02$ & .06 \\
\hline Pre-/postcentral gyrus WM & Right & $1.30 \pm 0.04$ & $1.27 \pm 0.04$ & .01 & $0.44 \pm 0.02$ & $0.48 \pm 0.03$ & $.00009^{\mathrm{a}}$ & $0.73 \pm 0.02$ & $0.74 \pm 0.02$ & .08 \\
\hline Parietal WM & Left & $1.31 \pm 0.05$ & $1.31 \pm 0.05$ & .94 & $0.57 \pm 0.04$ & $0.61 \pm 0.03$ & $.001^{\mathrm{a}}$ & $0.82 \pm 0.03$ & $0.84 \pm 0.03$ & .02 \\
\hline Parietal WM & Right & $1.29 \pm 0.06$ & $1.27 \pm 0.04$ & .28 & $0.58 \pm 0.03$ & $0.62 \pm 0.05$ & $.002^{\mathrm{a}}$ & $0.81 \pm 0.03$ & $0.84 \pm 0.04$ & .07 \\
\hline SLF & Left & $1.11 \pm 0.04$ & $1.09 \pm 0.03$ & .13 & $0.54 \pm 0.03$ & $0.57 \pm 0.04$ & .004 & $0.73 \pm 0.02$ & $0.75 \pm 0.03$ & .05 \\
\hline
\end{tabular}

Note:-HIV- indicates HIV-negative controls; HIV +, HIV-positive subjects.

a $P<.05 / 14 \approx .0035$ (Bonferroni-corrected for multiple comparisons). 

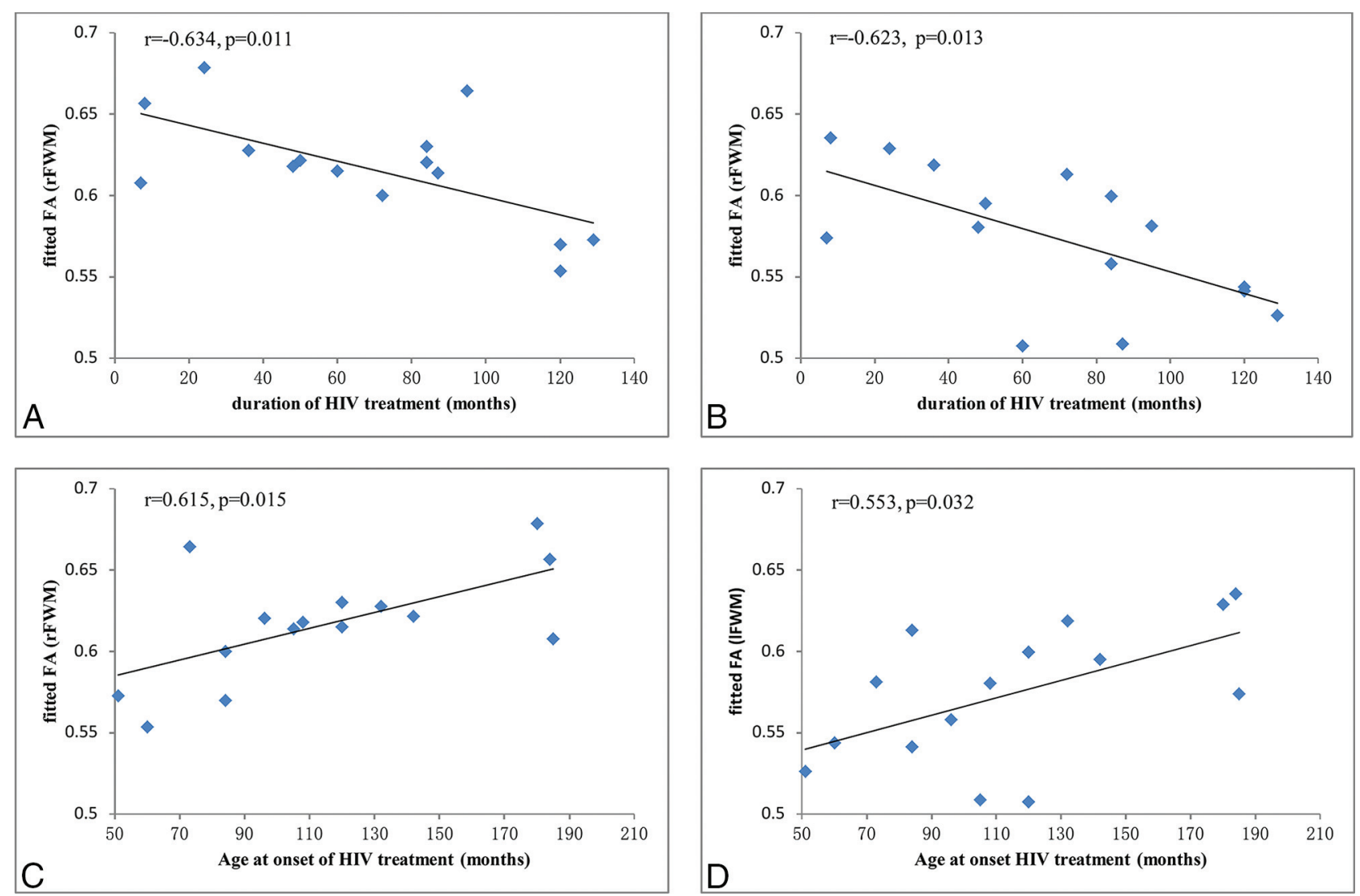

FIG 2. Correlation results between FA alterations and clinical measures in the HIV-positive subjects. $A$, FA values in the right frontal WM ( $r F W M)$ are negatively correlated with the duration of $\operatorname{HAART}(r=-0.634, P=.011) . B$, FA values in the left frontal WM (IFWM) are negatively correlated with the duration of HAART $(r=-0.623, P=.013)$. C, FA values in the rFWM are positively correlated with the age at onset of HAART $(r=0.615$, $P=.015)$. $D$, FA values in the IFWM are positively correlated with the age at onset of HAART $(r=0.553, P=.032)$.

the genu, body, and splenium of the corpus callosum were reduced in HIV-positive adolescents are consistent with findings in previous studies on HIV-infected children and adults. For example, FA values for the genu, ${ }^{10}$ body, ${ }^{21}$ and splenium ${ }^{8}$ of the corpus callosum $^{11,22}$ are significantly reduced in HIV-infected adults. Decreased FA of the body of corpus callosum is also reported in HIV-infected children. ${ }^{14}$ In addition, a lower FA in the genu of corpus callosum is demonstrated in a macaque model of neuroAIDS. ${ }^{23}$ Greater callosal maturation is associated with greater motor function. ${ }^{24}$ The corpus callosum is also involved with memory and executive function. ${ }^{25}$ Moreover, deceased FA is also found in the anterior and superior corona radiata in HIV-infected participants. ${ }^{10,11}$ The anterior corona radiata is associated with functions of the executive network. ${ }^{26}$ Previous studies have focused on HIV-infected children and found that the subjects displayed visual-spatial memory and motor developmental deficits and executive function disorders. ${ }^{27,28}$ Taken together, it can be concluded that a decline in the microstructural integrity of WM fibers may account for cognitive decline in HIV-positive adolescents.

In addition, patients with asymptomatic HIV have an abnormal WM microstructure. Xuan et $\mathrm{al}^{29}$ reported that the mean FA values were significantly lower and the mean AIDS dementia complex values were significantly higher in the corpus callosum and periventricular, frontal, and parietal WM in the asymptomatic group. A similar result has been found in the study by Hoare et al. ${ }^{14}$ These results imply that patients with HIV may have alter- ations in the diffusion of water molecules in their brain WM, whether they have symptoms or not. In our study, most of the subjects were also asymptomatic, and there were no significant differences in the Montreal Cognitive Assessment and Mini-Mental State Examination scores between the 2 groups.

The genu and splenium of the corpus callosum reach $90 \%$ of the maximum FA value by 11 years of age and demonstrate the earliest and most rapid FA changes with age, while the corona radiata demonstrates no FA changes with age from 5 to 30 years of age. ${ }^{30}$ Age-related FA increases in the adolescent group, including the body of the corpus callosum and right superior corona radiata. In contrast, in the young adult group, the FA changes are much less prominent. ${ }^{31}$ By $8-9$ months, the corpus callosum appears identical to that of an adult. ${ }^{32}$ All these results indicate that early HIV infection may affect WM development, especially in the corpus callosum and corona radiata.

Reduced FA is a well-established biomarker for the impaired integrity of WM. FA may be affected by many factors, including myelination, axon size and attenuation, path geometry, and extracellular water space between fibers. ${ }^{19}$ In our study, FA reduction in HIV-positive adolescents was mainly driven by an increase in $\mathrm{RD}$ (no changes in $\mathrm{AD}$ ). It is generally believed that $\mathrm{RD}$ mainly reflects the integrity and thickness of the myelin sheets covering the axons. ${ }^{33}$ Although the mechanism of this interesting phenomenon is not yet clear, we presume that it may be related to a manifestation of disrupted integrity of myelin or even hypomyelina- 
tion in the affected brain regions and the axonal injury cannot be identified. Related studies have demonstrated the predominant pathologic features, including pericapillary multinucleated giant cells, myelin loss, reactive astrocytosis, and microglial activation with microglial nodules. ${ }^{34}$ These factors may influence myelination and myelin development. The above findings indicate that the WM integrity may serve as a potential new treatment target for HIV-positive patients, and FA may be used as a qualified biomarker to understand the underlying mechanisms of injury or to evaluate the effectiveness of early interventions in adolescents with vertically transmitted HIV infections.

In this study, we found that the FA values in the bilateral frontal WM were negatively correlated with the duration of HAART and positively correlated with the age at onset of HAART. Although HAART can effectively suppress the HIV systemic burden, poor penetration into the CNS provides incomplete protection. Increasing evidence has also suggested that certain HAARTs may cause mitochondrial toxicity and lead to neuronal loss. ${ }^{16,35}$ This finding suggests that a longer HIV treatment may be associated with possible neurotoxicity in the WM of HIV-infected adolescents. However, the HIV treatment is offered to patients with $<350 \mathrm{CD} 4+\mathrm{T}$ cells $/ \mathrm{mm}^{3}$ or plasma HIV ribonucleic acid levels of $>55,000$ copies $/ \mathrm{mL},{ }^{36}$ so the earlier and longer HIV treatment may have been due to more virulent strains, thus accounting for direct viral effects on the WM by a very heterogeneous viral population. The early formation of myelin integrity may be disrupted irreversibly. In general, we infer that the cerebral WM development and myelination of HIV-infected adolescents might be affected by the HIV treatment and direct viral effects, especially in the frontal lobe.

In addition, the bilateral frontal white matter appears to be more involved according to the significant correlation results. Pomara et $\mathrm{al}^{37}$ found that subjects with HIV who were receiving HAART had significantly decreased FA compared with the healthy controls only in the frontal lobes. This is possibly because the frontal WM myelination may be more vulnerable during brain maturation.

There are several limitations in this study. First, cross-sectional and longitudinal studies on HIV-infected adolescents are needed to confirm the correlation between DTI alterations and neurocognitive performance, and more detailed neurocognitive tests should be performed. Furthermore, all of our subjects were receiving HAART during the DTI examination. Thus, we could not compare the potential differences between treated and untreated patients in the DTI analysis.

\section{CONCLUSIONS}

Adolescents with vertically transmitted HIV infections demonstrated microstructural WM damage as measured by reduced FA values in some brain regions, which may be caused by disrupted myelin integrity associated with increased RD. Our results have the potential to improve our understanding of the pathogenesis of brain WM changes in adolescents with vertically transmitted HIV infections and indicate that early HIV infection may affect WM development, especially in the frontal WM, corpus callosum, and corona radiata of adolescents. New neuroprotective regimens should be developed and performed earlier for children and ado- lescents with vertically transmitted HIV infections. The effectiveness of specific, early interventions can be confirmed by DTI, and FA values may be qualified biomarkers.

\section{REFERENCES}

1. Sullivan PS, Jones JS, Baral SD. The global north: HIV epidemiology in high-income countries. Curr Opin HIV AIDS 2014;9:199-205

2. Platzker AC, Colin AA, Chen XC, et al. Thoracoabdominal compression and respiratory system compliance in HIV-infected infants. Am J Respir Crit Care Med 2000;161:1567-71

3. Winter H. Gastrointestinal tract function and malnutrition in HIVinfected children. J Nutr 1996;126:2620S-22S

4. Price RW, Brew B, Sidtis J, et al. The brain in AIDS: central nervous system HIV-1 infection and AIDS dementia complex. Science 1988;239:586-92

5. Ragin AB, Storey P, Cohen BA, et al. Disease burden in HIV-associated cognitive impairment: a study of whole-brain imaging measures. Neurology 2004;63:2293-97

6. Basser PJ, Pierpaoli C. Microstructural and physiological features of tissues elucidated by quantitative-diffusion-tensor MRI. J Magn Reson B 1996;111:209-19

7. Filippi CG, Ulug AM, Ryan E, et al. Diffusion tensor imaging of patients with HIV and normal-appearing white matter on MR images of the brain. AJNR Am J Neuroradiol 2001;22:277-83

8. Wu Y, Storey P, Cohen BA, et al. Diffusion alterations in corpus callosum of patients with HIV. AJNR Am J Neuroradiol 2006;27: 656-60

9. Hoare J, Fouche JP, Spottiswoode B, et al. White-matter damage in Clade C HIV-positive subjects: a diffusion tensor imaging study. J Neuropsychiatry Clin Neurosci 2011;23:308-15

10. Zhu T, Zhong J, Hu R, et al. Patterns of white matter injury in HIV infection after partial immune reconstitution: a DTI tract-based spatial statistics study. J Neurovirol 2013;19:10-23

11. Nir TM, Jahanshad N, Busovaca E, et al. Mapping white matter integrity in elderly people with HIV. Hum Brain Mapp 2014;35:975-92

12. Chang L, Wong V, Nakama $\mathrm{H}$, et al. Greater than age-related changes in brain diffusion of HIV patients after 1 year. J Neuroimmune Pharmacol 2008;3:265-74

13. Sarma MK, Nagarajan R, Keller MA, et al. Regional brain gray and white matter changes in perinatally HIV-infected adolescents. $\mathrm{Neu}$ roimage Clin 2014;4:29-34

14. Hoare J, Fouche JP, Spottiswoode B, et al. A diffusion tensor imaging and neurocognitive study of HIV-positive children who are HAART- naïve "slow progressors." J Neurovirol 2012;18:205-12

15. Gongvatana A, Cohen RA, Correia S, et al. Clinical contributors to cerebral white matter integrity in $\mathrm{HIV}$-infected individuals. J Neurovirol 2011;17:477-86

16. Robertson KR, Su Z, Margolis DM, et al. Neurocognitive effects of treatment interruption in stable HIV-positive patients in an observational cohort. Neurology 2010;74:1260-66

17. Murphy DA, Sarr M, Durako SJ, et al. Barriers to HAART adherence among human immunodeficiency virus-infected adolescents. Arch Pediatr Adolesc Med 2003; 157:249-55

18. Auld AF, Agolory SG, Shiraishi RW, et al. Antiretroviral therapy enrollment characteristics and outcomes among HIV-infected adolescents and young adults compared with older adults: seven African countries, 2004-2013. MMWR Morb Mortal Wkly Rep 2014; 63:1097-103

19. Le Bihan D. Looking into the functional architecture of the brain with diffusion MRI. Nat Rev Neurosci 2003;4:469-80

20. Smith SM, Jenkinson M, Johansen-Berg H, et al. Tract-based spatial statistics: voxelwise analysis of multi-subject diffusion data. Neuroimage 2006;31:1487-505

21. Leite SC, Corrêa DG, Doring TM, et al. Diffusion tensor MRI evaluation of the corona radiata, cingulate gyri, and corpus callosum in HIV patients. J Magn Reson Imaging 2013;38:1488-93

22. Chen $\mathrm{Y}, \mathrm{An} \mathrm{H}, \mathrm{Zhu} \mathrm{H}$, et al. White matter abnormalities revealed by 
diffusion tensor imaging in non-demented and demented HIV+ patients. Neuroimage 2009;47:1154-62

23. Li C, Zhang X, Komery A, et al. Longitudinal diffusion tensor imaging and perfusion MRI investigation in a macaque model of neuroAIDS: a preliminary study. Neuroimage 2011;58:286-92

24. Mathew P, Pannek K, Snow P, et al. Maturation of corpus callosum anterior midbody is associated with neonatal motor function in eight preterm-born infants. Neural Plast 2013;2013:359532

25. Voineskos AN, Rajji TK, Lobaugh NJ, et al. Age-related decline in white matter tract integrity and cognitive performance: a DTI tractography and structural equation modeling study. Neurobiol Aging 2012;33:21-34

26. Yin $\mathrm{X}, \mathrm{Han} \mathrm{Y}, \mathrm{Ge} \mathrm{H}$, et al. Inferior frontal white matter asymmetry correlates with executive control of attention. Hum Brain Mapp 2013;34:796-813

27. Boivin MJ, Green SD, Davies AG, et al. A preliminary evaluation of the cognitive and motor effects of pediatric HIV infection in Zairian children. Health Psychol 1995;14:13-21

28. Nozyce ML, Lee SS, Wiznia A, et al. A behavioral and cognitive profile of clinically stable HIV-infected children. Pediatrics 2006;117:763-70

29. Xuan A, Wang GB, Shi DP, et al. Initial study of magnetic resonance diffusion tensor imaging in brain white matter of early AIDS patients. Chin Med J (Engl) 2013;126:2720-24

30. Lebel C, Walker L, Leemans A, et al. Microstructural maturation of the human brain from childhood to adulthood. Neuroimage 2008;40:1044-55

31. Giorgio A, Watkins KE, Douaud G, et al. Changes in white matter microstructure during adolescence. Neuroimage 2008;39:52-61

32. Barkovich AJ, Kjos BO. Normal postnatal development of the corpus callosum as demonstrated by MR imaging. AJNR Am J Neuroradiol 1988;9:487-91

33. Song SK, Sun SW, Ramsbottom MJ, et al. Dysmyelination revealed through MRI as increased radial (but unchanged axial) diffusion of water. Neuroimage 2002;17:1429-36

34. Smith AB, Smirniotopoulos JG, Rushing EJ. From the archives of the AFIP: central nervous system infections associated with human immunodeficiency virus infection: radiologic-pathologic correlation. Radiographics 2008;28:2033-58

35. Marra CM, Zhao Y, Clifford DB, et al; AIDS Clinical Trials Group 736 Study Team. Impact of combination antiretroviral therapy on cerebrospinal fluid HIV RNA and neurocognitive performance. AIDS 2009;23:1359-66

36. Dybul M, Fauci AS, Bartlett JG, et al; Panel on Clinical Practices for Treatment of HIV. Guidelines for using antiretroviral agents among HIV-infected adults and adolescents. Ann Intern Med 2002;137:381-433

37. Pomara N, Crandall DT, Choi SJ, et al. White matter abnormalities in HIV-1 infection: a diffusion tensor imaging study. Psychiatry Res 2001;106:15-24 